\title{
Contribution of hadronic $\gamma$-rays in the high energy spectra of black hole binaries
}

\section{S. Bhattacharyya*}

Astrophysical Sciences Division, Bhabha Atomic Research Centre, Mumbai-400085, India

E-mail: subirb@barc.ernet.in

\section{N. Bhatt}

Astrophysical Sciences Division, Bhabha Atomic Research Centre, Mumbai-400085, India

E-mail: nilayb@barc.ernet.in

\section{R. Misra}

Inter-University Centre for Astronomy and Astrophysics, post Bag 4, Ganeskhind, Pune-411007, India

E-mail: rmisra@iucaa.ernet.in

In the inner regions of an accretion disk around a black hole, relativistic protons can interact with ambient matter to produce electrons, positrons and $\gamma$-rays. The final steady state electron and positron spectrum are self-consistently calculated by solving an integro-differential equation where the Coulomb and Compton cooling of particles, $e^{ \pm}$pair production due to $\gamma-\gamma$ annihilation and pair annihilation are taken into account. The resultant $\gamma$-ray spectrum shows that the contribution of high energy photons $(E>0.8 \mathrm{MeV})$ could be entirely due to $p-p$ interactions. The observed X-ray/ $\gamma$-ray spectrum of Cyg X-1, in Hard and Soft states, are fitted with the present model. It is shown that GLAST will be able to detect black hole binaries and provide evidence for the presence of non-thermal protons in the accretion disk

VI Microquasar Workshop: Microquasars and Beyond

September 18-22 2006

Società del Casino, Como, Italy

\footnotetext{
*Speaker.
} 


\section{Introduction}

Black hole X-ray binaries (BHXRBs) are generally found in one of the two spectral states (a) hard state and $(b)$ soft state. In hard state the observed spectrum can be described by a hard power-law with spectral index $\Gamma \sim 1.7$ and an exponential cut-off at around $100 \mathrm{keV}$. Detailed modeling of the observed spectra reveals that it can be represented by thermal Comptonization of soft photons by a plasma having temperature $T \sim 100 \mathrm{keV}$ and optical depth of order unity [5, 3] . In soft state, the spectra consists of a blackbody-like component with $k T \sim 1 \mathrm{keV}$ and a steep power-law tail with no detectable cut-off upto $\sim 8 \mathrm{MeV}[11,14]$. The detail modeling of the spectrum shows that the it can be well fitted by a model in which soft photons are Comptonized by a plasma consisting of both thermal and non-thermal electron distributions in a hybrid state. Comptonization by thermal electrons $(k T \sim 1 \mathrm{keV})$ produce the blackbody-like component in the spectrum while the Comptonization by non-thermal electrons generate the non-thermal component of the observed spectrum. In this model, the steady state non-thermal electron distribution is selfconsistently calculated by assuming that there is an injection of non-thermal particles into the system, where they are cooled by Comptonization and Coulomb interactions [6].

One major uncertainity in the above mentioned Comptonization model is that it is not clear what kind of an electron acceleration mechanism is active in these sources. Whatever be the process, it will have to be highly efficient to produce non-thermal electrons in an environment where they cool rapidly by inverse Compton process. If the acceleration process is mass independent then protons are also expected to be accelerated to relativistic energies, for example, by scattering off magnetic 'kinks' in a Keplerian accretion disc [12] or by first order Fermi acceleration process in the shocks produced in the accretion flow [7]. Some of these non-thermal non-thermal protons may escape from the system and contribute to the jet formation [13]. This is interesting because there is some evidence that the X-ray producung region may be the same as the base of the extended jet [10]. Therefore the signature of these non-thermal protons will provide valuable information to the nature of black hole systems.

In the present work, we assume that the protons are accelerated to relativistic energies in the inner region of accretion disk forming a non-thermal spectrum. Relativistic protons undergo inelastic collision with thermal protons to produce charged and neutral pions, which, in turn, decay into electrons, positrons and $\gamma$-rays. These high energy electrons and positrons cool by inverse Comptonization of ambient photons and Coulomb cooling with the background thermal plasma. Annihilation of non-thermal positrons with ambient thermal elecrons is also taken into account. Steady state photon spectrum is calculated by considering the $\gamma-\gamma$ pair production. The subsequent effect of photon-photon pair production is also taken into account while calculating the spectra of high energy electron and positron. In this respect, this work differs from previous computation of spectra from non-thermal electron distributions because of pion decay, in which pair production due to the presence of copious soft photons are not considered $[4,8,9]$.

\section{Formalism}

We consider a uniform sphere of non-relativistic thermal plasma with number density $n_{T}$ and radius $R$, in the presence of an external copious photon source. It is convenient to param- 
eterize the luminosity of the external photons $L_{p h}$, in terms of the compactness parameter $l_{p h} \equiv$ $L_{p h} \sigma_{T} /\left(R m_{e} c^{3}\right)$, where $\sigma_{T}$ is the Thomson cross-section. The spectral shape of the ambient photons is taken either to be a Wien peak or an exponentially cutoff power-law depending on the spectral state being considered. In this system, we assume that there is a power-law distribution of non-thermal protons with index $\alpha$, which would lead to proton-proton collisions i.e. the density of non-thermal protons is given by

$$
n_{N T}(\gamma) d \gamma=n_{o}(\gamma-1)^{-\alpha} d \gamma
$$

The normalization $n_{o}$ of this distribution is characterized by the compactness parameter $l_{p-p} \equiv$ $L_{p-p} \sigma_{T} /\left(R m_{e} c^{3}\right)$, where $L_{p-p}$ is the total power in electron, positrons and $\gamma$-rays which would be produced in the proton-proton interactions.

The steady state positron density $N_{+}(\gamma)$ is determined by solving the integro-differential equation

$$
\frac{\partial}{\partial \gamma}\left(\dot{\gamma}_{C} N_{+}(\gamma)\right)+N_{+}(\gamma) \int_{1}^{\gamma} d \gamma^{\prime} P\left(\gamma, \gamma^{\prime}\right)-\int_{\gamma}^{\infty} d \gamma^{\prime} P\left(\gamma^{\prime}, \gamma\right) N_{+}\left(\gamma^{\prime}\right)+\dot{N}_{+}(\gamma)=Q_{+, p p}(\gamma)+Q_{+, \gamma \gamma}(\gamma)
$$

while the corresponding electron density $N_{-}(\gamma)$ is obtained from

$$
\frac{\partial}{\partial \gamma}\left(\dot{\gamma}_{C} N_{-}(\gamma)\right)+N_{-}(\gamma) \int_{1}^{\gamma} d \gamma^{\prime} P\left(\gamma, \gamma^{\prime}\right)-\int_{\gamma}^{\infty} d \gamma^{\prime} P\left(\gamma^{\prime}, \gamma\right) N_{-}\left(\gamma^{\prime}\right)=Q_{-, p p}(\gamma)+Q_{-, \gamma \gamma}(\gamma)
$$

Here, $P(\gamma, \gamma-e) d e d t$ is the probability that a positron/electron with Lorentz factor $\gamma$ will suffer a collision and its Lorentz factor changes by an amount between $e$ and $e+d e$ in time $d t$, where $e \equiv h v / m_{e} c^{2}$ is the normalized photon energy. $Q_{ \pm, \gamma \gamma}$ and $Q_{ \pm, p p}$ are the creation rates of pairs due to $p-p$ interactions and photon-photon production respectively, $\dot{N}_{+}(\gamma)$ is the annihilation rates of positrons with the thermal background electrons and $\dot{\gamma}_{C}$ is the Coulomb cooling rate. This formalism to obtain the particle distribution follows from the results described in [2] (and references therein), where the exact expression for $P(\gamma, \gamma-e) d e d t$ has been derived. For small changes in particle energy, the integral terms in equations (2.2) and (2.3) can be reduced to $\frac{\partial}{\partial \gamma}\left[\dot{\gamma}_{I C} N_{ \pm}(\gamma)\right]$ where $\dot{\gamma}_{I C}$ is the inverse Compton cooling rate. It was these diffusion equations that were used by [1] as a simplifying assumption. The equilibrium photon density inside the sphere is a solution of

$$
Q_{\gamma, I C}+Q_{\gamma, p p}+Q_{\gamma, e^{+} e^{-}}=n_{\gamma}(e)\left[R_{\gamma \gamma}+\frac{c}{R\left[1+\tau_{K N}(e)\right]}\right]
$$

where $R_{\gamma \gamma}(e)$ is the rate of photon annihilation and $\tau_{K N}$ is the Klein-Nishina optical depth. $Q_{\gamma, I C}$, $Q_{\gamma, p p}$ and $Q_{\gamma, e^{+} e^{-}}$are the photon production rates due to inverse Compton, $p-p$ interaction and pair annihilation respectively. Equations (2.2), (2.3) and (2.4) are solved self-consistently to obtain electron and positron distributions as well as the radiative flux. The output spectrum depends on the spectral shape of the ambient photons and three other parameters: the Thomson optical depth $\tau$, photon compactness $l_{p h}$ and the ratio of proton-proton compactness $l_{p-p}$, to that of the ambient photon, $\beta \equiv l_{p-p} / l_{p h}$. The results are insensitive to the non-thermal proton index $\alpha$ and the size $R$ of the system. 

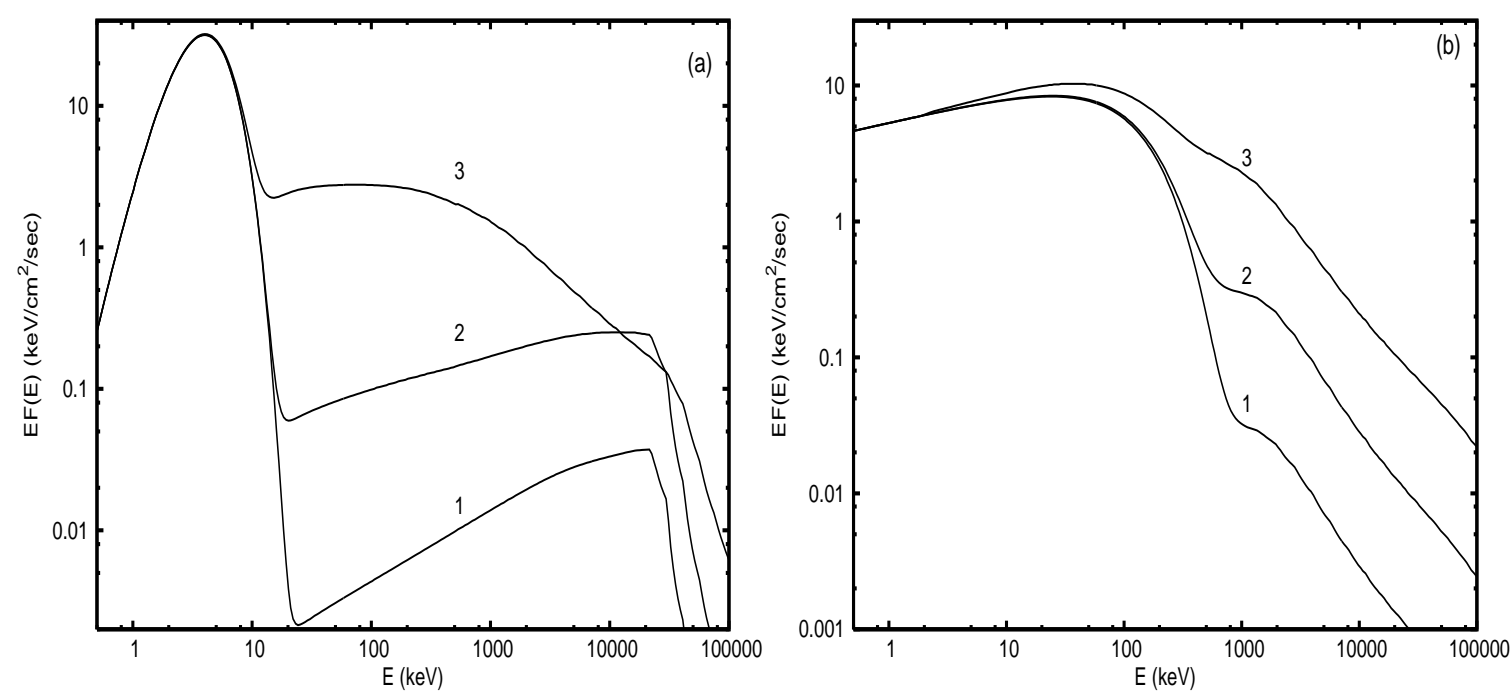

Figure 1: The radiated spectra for parameters typical of $(a)$ soft state of black hole binaries, which are $l_{p h}=250, k T_{p h}=1 \mathrm{keV}, \tau=2.5$ and $\alpha=2.5$ and $(b)$ hard state of black hole binaries, which are $l_{p h}=250$, $E_{c}=100 \mathrm{keV}, \tau=1.0$ and $\alpha=2.5$. For both the plots, three curves labeled as 1,2 and 3 are for compactness ratio $\beta \equiv l_{p-p} / l_{p h}=0.001,0.01$ and 0.1 respectively.

\section{Results and Discussion}

The computed radiated spectra due to p-p interactions, corresponding to parameters typical to soft and hard states of black hole binaries are shown in Figs. 1 and 2 respectively. The main motivation here is to investigate the contribution of p-p interaction to $\gamma$-ray part of the spectrum. Therefore a detailed fit to the broad-band data is not being attempted.In the soft state, dominant soft component is being represented by a Wien peak like spectrum with $k T_{p h}=1 \mathrm{keV}$ and high compactness, $l_{p h}=250$. The corresponding luminosity is $L \sim 10^{38}\left(\frac{l_{p h}}{250}\right)\left(\frac{R}{10^{7} \mathrm{~cm}}\right) \mathrm{erg} \mathrm{s}^{-1}$ where $R=10^{7} \mathrm{~cm}$ is the size of the system. The optical depth of the thermal electron is of order unity. The spectra plotted in Fig. 1 correspond to three different values of the compactness ratio $\beta=0.001$, 0.01 and 0.1 which represent increasing efficiency of the acceleration process to produce nonthermal protons. At low $\beta$ values the spectra are hard with a break around $\sim 80 \mathrm{MeV}$. This cutoff is due to the interaction of higher energy photons with the ambient soft photons of energy $\sim 3$ $\mathrm{keV}$ to generate pairs. For higher compactness ratio, $\beta=0.01$, pair creation due to photon-photon interaction affects the particle distribution at low $\gamma$, which leads to a softer output spectra. For still larger values of $\beta=0.1$, another break at around $511 \mathrm{keV}$ appears in the radiated spectrum, since the density of high energy photons is large enough for pairs to be produced by self interaction. The spectra for hard state are also computed for the same values of $\beta$ as in the soft state when the ambient photon density is similar to that found in the hard state of black hole binaries. In this state, the spectrum is dominated by a thermal Comptonized component, which is approximated here as an exponentially cut off power law with photon index $\Gamma=1.7$ and cut off energy $E_{c}=100 \mathrm{keV}$. As the average photon energy in the plasma is higher than that of the soft state, the cut-off in the photon spectrum due to pair production appears at $\approx 3 \mathrm{MeV}$ instead of $\approx 80 \mathrm{MeV}$. Since the number density of ambient photons with energy $>500 \mathrm{keV}$ is large, pair production is dominated by interaction 

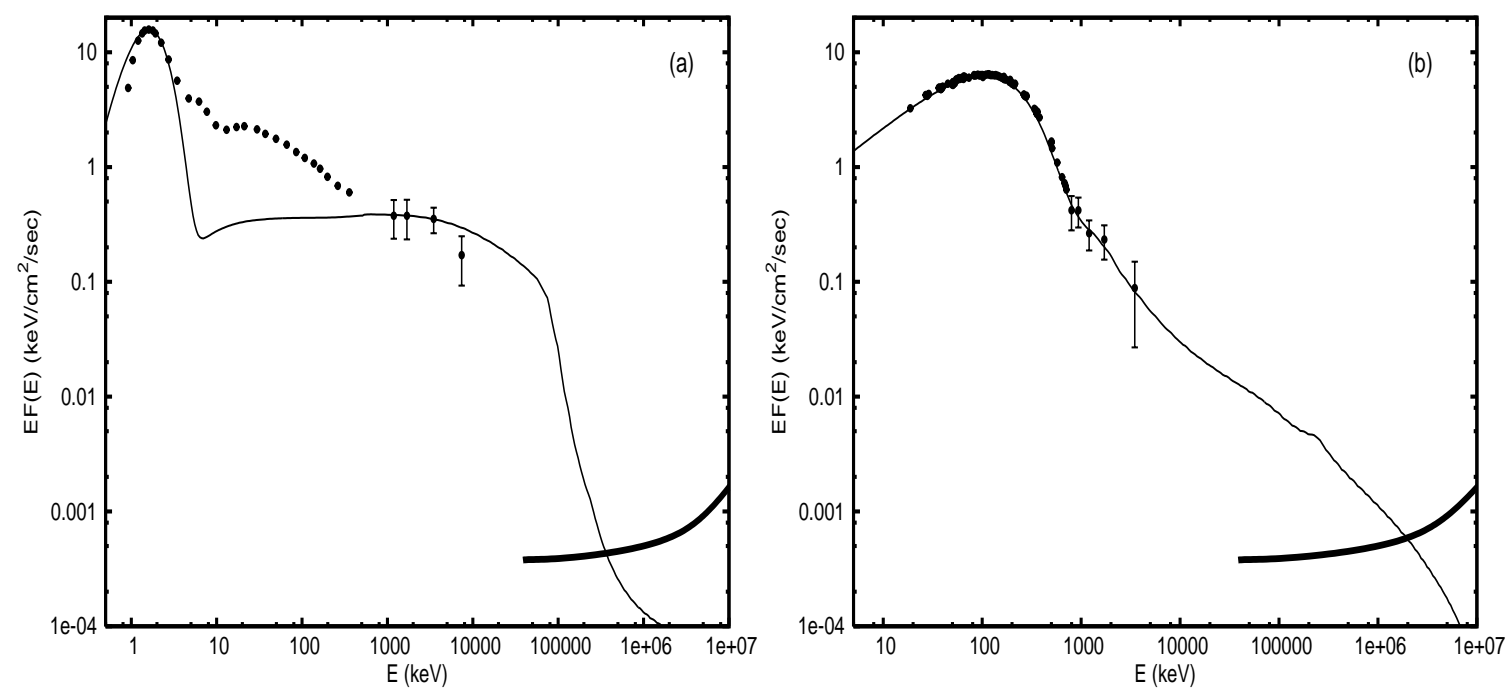

Figure 2: (a) Broad band soft state data of Cyg X-1 reproduced from [11]. Solid line is the computed spectrum for $\beta=0.046, l_{p h}=125, k T_{p h}=0.4 \mathrm{keV}, \tau=2.5$ and $\alpha=2.5$. (b) Broad band hard state data of Cyg X-1 reproduced from [11]. Solid line is computed spectrum for $\beta=0.018, l_{p h}=110, E_{c}=120 \mathrm{keV}$, $\tau=1.0$ and $\alpha=2.5$. The thick broad line in both plots shows the estimated GLAST sensitivity (http://wwwglast.slac.stanford.edu/software/IS/glast_lati_performance.htm).

of non-thermal photons with the background ambient ones even for large compactness. Thus, the shape of the computed spectra is relatively invariant to $\beta$ as shown in Figure 2 and in particular the slope of the expected spectrum at energies $>\mathrm{MeV}$ is insensitive to the parameters of the model.

The comparison of the computed specra with broad band observed data in soft and hard state of Cyg X-1 are shown in Figs 3 and 4. The hard x-ray spectrum $(3-800 \mathrm{keV})$ of the soft state are too steep to be explained as emission due to p-p interactions. But the observed $\gamma$-ray spectra in $0.8-8 \mathrm{MeV}$ energy range could be due to such interactions. In case of hard state the observed $\gamma-$ ray spectrum $(0.5-8 \mathrm{MeV})$ can be explained as emission due to $p-p$ interaction alone. However, the model predicts that for a reasonable parameters, GLAST should be able to detect black hole binaries.

\section{Conclusions}

A self-consistent scheme, which computes the electron/positron and radiation energy distribution inside a thermal plasma having non-thermal protons, is developed. The non-thermal protons interact via $p-p$ collisions to produce electron/positron pairs and $\gamma$-rays. These high energy pairs cool by inverse Comptonization of ambient photons. The effect of subsequent pair production due to photon-photon interactions and annihilation of positrons with ambient electrons, is taken into account. Comparison of the computed spectra with the broad band X-ray/ $\gamma$-ray spectra of black hole binaries in different spectral states reveal:

- The hard X-ray spectra (3-800 keV) of the soft and Very High state (VHS), are too steep to be explained as emission due to $p-p$ interactions. However, the observed $\gamma$-ray spectra $(0.8-8 \mathrm{MeV})$, especially for the soft state, could be due to such interactions. 
- For the hard state, the observed $\gamma$-ray spectrum (0.5-8 MeV) can be explained as emission due to $p-p$ interactions alone. The predicted steep spectral shape in this energy range, is not sensitive to the model parameters, and matches well with the observations.

- For both the soft and hard states, the model predicts that for reasonable parameters, GLAST should be able to detect black hole binaries. This in contrast to the situation when only non-thermal electrons are present in the system, where very low compactness and large maximum Lorentz factor of the electrons have to be postulated, in order for GLAST to make a similar detection.

\section{References}

[1] Bhattacharyya, S., Bhatt, N., Misra, R., Kaul, C. L. 2003, ApJ, 595, 317

[2] Blumenthal, G. R., Gould, R. J. 1970, Rev. Mod. Phys. 42, 237

[3] Del Santo, M., et al astro-ph/0611107

[4] Eilek, J. A., Kafatos, M., 1983, ApJ, 271, 804

[5] Gierliński, M., Zdziarski A. A., Done, C., Johnson W. N., Ebisawa K., Ueda, Y., Haardt, F. Phlips, B. F. 1997, MNRAS, 288, 958

[6] Gierliński, M., Zdziarski A. A., Poutanen J., Coppi P. S., Ebisawa, K., Johnson W. N. 1999, MNRAS, 309, 496

[7] Le, T., Becker, P, A. 2005, ApJ, 632, 476

[8] Mahadevan, R. Narayan, R., Krolik, J. 1997, ApJ, 486, 268

[9] Markoff, S., Melia, F., Sarcevic, I. 1999, ApJ, 522, 870

[10] Markoff, S., Nowak, M. A., Wilms, J. 2005, ApJ, 635, 1203

[11] McConnell, M. L., et al. 2002, ApJ, 572, 984

[12] Subramanian, P., Becker, P.A., Kafatos, M. 1996, ApJ, 469, 784

[13] Subramanian, P., Becker, P. A., Kazanas, D. 1999, ApJ, 523, 203

[14] Zdziarski, A. A. Grove, J. E., Poutanen, J., Rao, A. R., Vadawale, S. V. 2001, ApJ, 554, L45 